\title{
Research Article \\ Strong Convergence of Viscosity Iteration Methods for Nonexpansive Mappings
}

\author{
Jong Soo Jung \\ Department of Mathematics, Dong-A University, Busan 604-714, South Korea \\ Correspondence should be addressed to Jong Soo Jung, jungjs@mail.donga.ac.kr
}

Received 29 December 2008; Accepted 7 March 2009

Recommended by Simeon Reich

We propose a new viscosity iterative scheme for finding fixed points of nonexpansive mappings in a reflexive Banach space having a uniformly Gâteaux differentiable norm and satisfying that every weakly compact convex subset of the space has the fixed point property for nonexpansive mappings. Certain different control conditions for viscosity iterative scheme are given and strong convergence of viscosity iterative scheme to a solution of a ceratin variational inequality is established.

Copyright (C 2009 Jong Soo Jung. This is an open access article distributed under the Creative Commons Attribution License, which permits unrestricted use, distribution, and reproduction in any medium, provided the original work is properly cited.

\section{Introduction}

Let $E$ be a real Banach space and let $C$ be a nonempty closed convex subset of $E$. Recall that a mapping $f: C \rightarrow C$ is a contraction on $C$ if there exists a constant $k \in(0,1)$ such that $\|f(x)-f(y)\| \leq k\|x-y\|, x, y \in C$. We use $\Sigma_{C}$ to denote the collection of mappings $f$ verifying the above inequality. That is, $\Sigma_{C}=\{f: C \rightarrow C \mid f$ is a contraction with constant $k\}$. Let $T: C \rightarrow C$ be a nonexpansive mapping (recall that a mapping $T: C \rightarrow C$ is nonexpansive if $\|T x-T y\| \leq\|x-y\|, x, y \in C)$ and let $F(T)$ denote the set of fixed points of $T$; that is, $F(T)=\{x \in C: x=T x\}$. $(0,1)$,

We consider the iterative scheme: for $T$ a nonexpansive mapping, $f \in \Sigma_{\mathrm{C}}$ and $\alpha_{n} \in$

$$
x_{n+1}=\alpha_{n} f\left(x_{n}\right)+\left(1-\alpha_{n}\right) T x_{n}, \quad n \geq 0 .
$$

As a special case of (1.1), the following iterative scheme:

$$
z_{n+1}=\alpha_{n} u+\left(1-\alpha_{n}\right) T z_{n}, \quad n \geq 0,
$$


where $u, z_{0} \in C$ are arbitrary (but fixed), has been investigated by many authors; see, for example, Browder [1], Chang [2], Cho et al. [3], Halpern [4], Lions [5], Reich [6, 7], Shioji and Takahashi [8], Wittmann [9], and Xu [10]. The authors above showed that the sequence $\left\{z_{n}\right\}$ generated by (1.2) converges strongly to a point in the fixed-point set $F(T)$ under appropriate conditions on $\left\{\alpha_{n}\right\}$ in Hilbert spaces or certain Banach spaces.

The viscosity approximation method of selecting a particular fixed point of a given nonexpansive mapping in a Hilbert space was proposed by Moudafi [11] in 2000. In 2004, Xu [12] extended Theorem 2.2 of Moudafi [11] for the iterative scheme (1.1) to a Banach space setting by using the following conditions on the sequence $\left\{\alpha_{n}\right\}$ :

$$
\lim _{\mathrm{n} \rightarrow \infty} \alpha_{n}=0, \quad \sum_{n=0}^{\infty} \alpha_{n}=\infty, \quad \sum_{n=0}^{\infty}\left|\alpha_{n+1}-\alpha_{n}\right|<\infty \quad \text { or } \quad \lim _{n \rightarrow \infty} \frac{\alpha_{\mathrm{n}}}{\alpha_{n+1}}=1
$$

For the iterative scheme (1.1) with generalized contractive mappings instead of contractions, we refer to [13].

In 2005, Kim and Xu [14] provided a simpler modification of Mann iterative scheme in a uniformly smooth Banach space as follows:

$$
\begin{aligned}
x_{0} & =x \in C, \\
y_{n} & =\beta_{n} x_{n}+\left(1-\beta_{n}\right) T x_{n}, \\
x_{n+1} & =\alpha_{n} u+\left(1-\alpha_{n}\right) y_{n}, \quad n \geq 0,
\end{aligned}
$$

where $u \in C$ is an arbitrary (but fixed) element, and $\left\{\alpha_{n}\right\}$ and $\left\{\beta_{n}\right\}$ are two sequences in $(0,1)$. They proved that the sequence $\left\{x_{n}\right\}$ generated by (1.4) converges to a fixed point of $T$ under the following control conditions:

(i) $\lim _{n \rightarrow \infty} \alpha_{n}=0, \lim _{n \rightarrow \infty} \beta_{n}=0$;

(ii) $\sum_{n=0}^{\infty} \alpha_{n}=\infty, \sum_{n=0}^{\infty} \beta_{n}=\infty$;

(iii) $\sum_{n=0}^{\infty}\left|\alpha_{n+1}-\alpha_{n}\right|<\infty, \sum_{n=0}^{\infty}\left|\beta_{n+1}-\beta_{n}\right|<\infty$.

Recently, Yao et al. [15] considered the following modified Mann iterative scheme in a uniformly smooth Banach space as the viscosity approximation method:

$$
\begin{aligned}
x_{0} & =x \in C, \\
y_{n} & =\beta_{n} x_{n}+\left(1-\beta_{n}\right) T x_{n}, \\
x_{n+1} & =\alpha_{n} f\left(x_{n}\right)+\left(1-\alpha_{n}\right) y_{n}, \quad n \geq 0,
\end{aligned}
$$

and proved strong convergence of the sequence $\left\{x_{n}\right\}$ generated by (1.5) under certain different control conditions on $\left\{\alpha_{n}\right\}$ and $\left\{\beta_{n}\right\}$. In particular, their results remove the condition $\sum_{n=0}^{\infty}\left|\alpha_{n+1}-\alpha_{n}\right|<\infty$ imposed on $\left\{\alpha_{n}\right\}$. 
Very recently, Qin et al. [16] proposed the composite Halpern type iterative scheme in a uniformly smooth Banach space as follows:

$$
\begin{aligned}
x_{0} & =x, \quad u \in C, \\
z_{n} & =\gamma_{n} x_{n}+\left(1-\gamma_{n}\right) T x_{n}, \\
y_{n} & =\beta_{n} x_{n}+\left(1-\beta_{n}\right) T z_{n}, \\
x_{n+1} & =\alpha_{n} u+\left(1-\alpha_{n}\right) y_{n}, \quad n \geq 0,
\end{aligned}
$$

and showed strong convergence of the sequence $\left\{x_{n}\right\}$ generated by (1.6) under the following control conditions:

(i) $\sum_{n=0}^{\infty} \alpha_{n}=\infty$;

(ii) $\lim _{n \rightarrow \infty} \alpha_{n}=0, \lim _{n \rightarrow \infty} \beta_{n}=0$ and $0<a \leq \gamma_{n}$ for some $a \in(0,1)$;

(iii) $\sum_{n=0}^{\infty}\left|\alpha_{n+1}-\alpha_{n}\right|<\infty, \sum_{n=0}^{\infty}\left|\beta_{n+1}-\beta_{n}\right|<\infty, \sum_{n=0}^{\infty}\left|\gamma_{n+1}-\gamma_{n}\right|<\infty$.

In this paper, under the framework of a reflexive Banach space having a uniformly Gâteaux differentiable norm and satisfying that every weakly compact convex subset of $E$ has the fixed point property for nonexpansive mappings, we consider a new composite iterative scheme for a nonexpansive mapping $T$ as the viscosity approximation method: for $f \in \Sigma_{C}$ and the initial guess $x_{0}=x \in C$,

$$
\begin{aligned}
z_{n} & =\gamma_{n} x_{n}+\left(1-\gamma_{n}\right) T x_{n}, \\
y_{n} & =\beta_{n} x_{n}+\left(1-\beta_{n}\right) T z_{n}, \\
x_{n+1} & =\alpha_{n} f\left(x_{n}\right)+\left(1-\alpha_{n}\right) y_{n}, \quad n \geq 0,
\end{aligned}
$$

where $\left\{\alpha_{n}\right\},\left\{\beta_{n}\right\}$ and $\left\{\gamma_{n}\right\}$ are sequences in $(0,1)$. First, we prove under certain control conditions on the sequences $\left\{\alpha_{n}\right\},\left\{\beta_{n}\right\}$ and $\left\{\gamma_{n}\right\}$ different from those of Qin et al. [16] that the sequence $\left\{x_{n}\right\}$ generated by (IS) converges strongly to a fixed point of $T$, which is a solution of a certain variational inequality. Next we study the composite iterative scheme (IS) with the weakly contractive mapping instead of the contractions. The main results develop and complement the corresponding results of $[2,3,8,9,11,12,15,16]$. In particular, if $\beta_{n}=0$ for all $n \geq 0$ in (IS), then (IS) reduces a new viscosity iterative scheme for finding a fixed point of $T$ :

$$
\begin{aligned}
z_{n} & =\gamma_{n} x_{n}+\left(1-\gamma_{n}\right) T x_{n}, \\
x_{n+1} & =\alpha_{n} f\left(x_{n}\right)+\left(1-\alpha_{n}\right) T z_{n}, \quad n \geq 0 .
\end{aligned}
$$

\section{Preliminaries and Lemmas}

Let $E$ be a real Banach space with norm $\|\cdot\|$, and let $E^{*}$ be its dual. The value of $f \in E^{*}$ at $x \in E$ will be denoted by $\langle x, f\rangle$. When $\left\{x_{n}\right\}$ is a sequence in $E$, then $x_{n} \rightarrow x$ (resp., $x_{n} \rightarrow x$ ) will denote strong (resp., weak) convergence of the sequence $\left\{x_{n}\right\}$ to $x$. 
The (normalized) duality mapping $J$ from $E$ into the family of nonempty (by HahnBanach theorem) weak-star compact subsets of its dual $E^{*}$ is defined by

$$
J(x)=\left\{f \in E^{*}:\langle x, f\rangle=\|x\|^{2}=\|f\|^{2}\right\},
$$

for each $x \in E$ [17].

The norm of $E$ is said to be Gateaux differentiable (and $E$ is said to be smooth) if

$$
\lim _{t \rightarrow 0} \frac{\|x+t y\|-\|x\|}{t}
$$

exists for each $x, y$ in its unit sphere $U=\{x \in E:\|x\|=1\}$. The norm is said to be uniformly Gâteaux differentiable if for $y \in U$, the limit is attained uniformly for $x \in U$. The space $E$ is said to have a uniformly Fréchet differentiable norm (and $E$ is said to be uniformly smooth) if the limit in (2.2) is attained uniformly for $(x, y) \in U \times U$. It is known that $E$ is smooth if and only if each duality mapping $J$ is single-valued. It is also well-known that if $E$ has a uniformly Gâteaux differentiable norm, $J$ is uniformly norm-to-weak ${ }^{*}$ continuous on each bounded subsets of $E$ [17].

Let $C$ be a nonempty closed convex subset of $E$. $C$ is said to have the fixed point property for nonexpansive mappings if every nonexpansive mapping of a bounded closed convex subset $D$ of $C$ has a fixed point in $D$. Let $D$ be a subset of $C$. Then a mapping $Q: C \rightarrow D$ is said to be a retraction from $C$ onto $D$ if $Q x=x$ for all $x \in D$. A retraction $Q: C \rightarrow D$ is said to be sunny if $Q(Q x+t(x-Q x))=Q x$ for all $x \in C$ and $t \geq 0$ with $Q x+t(x-Q x) \in C$. A subset $D$ of $C$ is said to be a sunny nonexpansive retract of $C$ if there exists a sunny nonexpansive retraction of $C$ onto $D$. In a smooth Banach space $E$, it is well-known [18, page 48] that $Q$ is a sunny nonexpansive retraction from $C$ onto $D$ if and only if the following condition holds

$$
\langle x-Q x, J(z-Q x)\rangle \leq 0, \quad x \in C, z \in D .
$$

We need the following lemmas for the proof of our main results. Lemma 2.1 was also given in Jung and Morales [19], Lemma 2.2 is Lemma 2 of Suzuki [20] and Lemma 2.3 is essentially Lemma 2 of Liu [21] (also see [10]).

Lemma 2.1. Let $E$ be a real Banach space and let $J$ be the duality mapping. Then, for any given $x, y \in E$, one has

$$
\|x+y\|^{2} \leq\|x\|^{2}+2\langle y, j(x+y)\rangle
$$

for all $j(x+y) \in J(x+y)$.

Lemma 2.2. Let $\left\{x_{n}\right\}$ and $\left\{w_{n}\right\}$ be bounded sequences in a Banach space $E$ and let $\left\{\delta_{n}\right\}$ be a sequence in $[0,1]$ which satisfies the following condition:

$$
0<\liminf _{n \rightarrow \infty} \delta_{n} \leq \limsup _{n \rightarrow \infty} \delta_{n}<1 .
$$


suppose that

$$
\begin{gathered}
x_{n+1}=\delta_{n} x_{n}+\left(1-\delta_{n}\right) w_{n}, \quad n \geq 0, \\
\limsup _{n \rightarrow \infty}\left(\left\|w_{n+1}-w_{n}\right\|-\left\|x_{n+1}-x_{n}\right\|\right) \leq 0 .
\end{gathered}
$$

Then $\lim _{n \rightarrow \infty}\left\|w_{n}-x_{n}\right\|=0$.

Lemma 2.3. Let $\left\{s_{n}\right\}$ be a sequence of nonnegative real numbers satisfying

$$
s_{n+1} \leq\left(1-\lambda_{n}\right) s_{n}+\lambda_{n} \gamma_{n}+\delta_{n}, \quad n \geq 0,
$$

where $\left\{\lambda_{n}\right\},\left\{\gamma_{n}\right\}$, and $\left\{\delta_{n}\right\}$ satisfy the following conditions:

(i) $\left\{\lambda_{n}\right\} \subset[0,1]$ and $\sum_{n=0}^{\infty} \lambda_{n}=\infty$ or, equivalently, $\prod_{n=0}^{\infty}\left(1-\lambda_{n}\right)=0$;

(ii) $\lim \sup _{n \rightarrow \infty} \gamma_{n} \leq 0$ or $\sum_{n=1}^{\infty} \lambda_{n} \gamma_{n}<\infty$;

(iii) $\delta_{n} \geq 0 \quad(n \geq 0), \sum_{n=0}^{\infty} \delta_{n}<\infty$.

Then $\lim _{n \rightarrow \infty} s_{n}=0$.

Recall that a mapping $A: C \rightarrow C$ is said to be weakly contractive if

$$
\|A x-A y\| \leq\|x-y\|-\psi(\|x-y\|), \quad \forall x, y \in C,
$$

where $\psi:[0,+\infty) \rightarrow[0,+\infty)$ is a continuous and strictly increasing function such that $\psi$ is positive on $(0, \infty)$ and $\psi(0)=0$. As a special case, if $\psi(t)=(1-k) t$ for $t \in[0,+\infty)$, where $k \in(0,1)$, then the weakly contractive mapping $A$ is a contraction with constant $k$. Rhoades [22] obtained the following result for weakly contractive mapping.

Lemma 2.4 ([22, Theorem 2]). Let $(X, d)$ be a complete metric space and let $A$ be a weakly contractive mapping on $X$. Then $A$ has a unique fixed point $p$ in $X$. Moreover, for $x \in X,\left\{A^{n} x\right\}$ converges strongly to $p$.

The following Lemma was given in $[23,24]$.

Lemma 2.5. Let $\left\{s_{n}\right\}$ and $\left\{\gamma_{n}\right\}$ be two sequences of nonnegative real numbers and let $\left\{\lambda_{n}\right\}$ be a sequence of positive numbers satisfying the conditions:

(i) $\sum_{n=0}^{\infty} \lambda_{n}=\infty$;

(ii) $\lim _{n \rightarrow \infty}\left(\gamma_{n} / \lambda_{n}\right)=0$.

Let the recursive inequality,

$$
s_{n+1} \leq s_{n}-\lambda_{n} \psi\left(s_{n}\right)+\gamma_{n}, \quad n \geq 0,
$$


be given, where $\psi(t)$ is a continuous and strict increasing function on $[0,+\infty)$ with $\psi(0)=0$. Then $\lim _{n \rightarrow \infty} s_{n}=0$.

\section{Main Results}

First, we study a strong convergence theorem for a viscosity iterative scheme for the nonexpansive mapping with the contraction.

For $T: C \rightarrow C$ a nonexpansive mapping, $t \in(0,1)$ and $f \in \Sigma_{C}, t f+(1-t) T: C \rightarrow C$ defines a strict contraction mapping. Thus, by the Banach contraction mapping principle, there exists a unique fixed point $x_{t}^{f}$ satisfying

$$
x_{t}^{f}=t f\left(x_{t}^{f}\right)+(1-t) T x_{t}^{f} .
$$

For simplicity we will write $x_{t}$ for $x_{t}^{f}$ provided no confusion occurs.

In 2006, the following result was given by Jung [25] (see also Xu [12] for the result in uniformly smooth Banach spaces).

Theorem J (see [25]). Let E be a reflexive Banach space having a uniformly Gâteaux differentiable norm. Suppose that every weakly compact convex subset of $E$ has the fixed point property for nonexpansive mappings. Let $C$ be a nonempty closed convex subset of $E$ and let $T$ be a nonexpansive mapping from $C$ into itself with $F(T) \neq \emptyset$. Then $\left\{x_{t}\right\}$ defined by $(\mathrm{R})$ converges strongly to a point in $F(T)$. If we define $Q: \Sigma_{C} \rightarrow F(T)$ by

$$
Q(f):=\lim _{t \rightarrow 0^{+}} x_{t}, \quad f \in \Sigma_{C},
$$

then $Q(f)$ is the unique solution of the variational inequality

$$
\langle(I-f)(Q(f)), J(Q(f)-p)\rangle \leq 0, \quad f \in \Sigma_{C}, p \in F(T) .
$$

Remark 3.1. In Theorem J, if $f(x)=u \in C$ is a constant, then (VI) become

$$
\langle Q u-u, J(Q u-p)\rangle \leq 0, \quad u \in C, \quad p \in F(T)
$$

Hence by (2.3), $Q$ reduces to the sunny nonexpansive retraction from $C$ to $F(T)$. Namely $F(T)$ is a sunny nonexpansive retraction of $C$.

Using Theorem J, we have the following result.

Theorem 3.2. Let E be a reflexive Banach space having a uniformly Gâteaux differentiable norm. Suppose that every weakly compact convex subset of $E$ has the fixed point property for nonexpansive mappings. Let $C$ be a nonempty closed convex subset of $E$ and let $T$ be a nonexpansive mapping from $C$ 
into itself with $F(T) \neq \emptyset$. Let $\left\{\alpha_{n}\right\},\left\{\beta_{n}\right\}$, and $\left\{\gamma_{n}\right\}$ be sequences in $(0,1)$ which satisfy the conditions:

(C1) $\lim _{n \rightarrow \infty} \alpha_{n}=0, \sum_{n=0}^{\infty} \alpha_{n}=\infty$;

(C2) $\lim _{n \rightarrow \infty} \beta_{n}=0$;

(C3) $0<\liminf _{n \rightarrow \infty} \gamma_{n} \leq \lim \sup _{n \rightarrow \infty} \gamma_{n}<1$.

Let $f \in \Sigma_{C}$ and the initial guess $x_{0}=x \in C$ be chosen arbitrarily. Let $\left\{x_{n}\right\}$ be the sequence generated by

$$
\begin{aligned}
z_{n} & =\gamma_{n} x_{n}+\left(1-\gamma_{n}\right) T x_{n}, \\
y_{n} & =\beta_{n} x_{n}+\left(1-\beta_{n}\right) T z_{n}, \\
x_{n+1} & =\alpha_{n} f\left(x_{n}\right)+\left(1-\alpha_{n}\right) y_{n}, \quad n \geq 0 .
\end{aligned}
$$

If $\lim _{n \rightarrow \infty}\left\|T z_{n}-z_{n}\right\|=0$, then $\left\{x_{n}\right\}$ converges strongly to $Q(f) \in F(T)$, where $Q(f)$ is the unique solution of the variational inequality

$$
\langle(I-f)(Q(f)), J(Q(f)-p)\rangle \leq 0, \quad f \in \Sigma_{C}, p \in F(T) .
$$

Proof. We note that by Theorem J, there exists the unique solution $Q(f)$ of the variational inequality

$$
\langle(I-f)(Q(f)), J(Q(f)-p)\rangle \leq 0, \quad f \in \Sigma_{C}, p \in F(T) .
$$

Namely, $Q(f)=\lim _{t \rightarrow 0^{+}} x_{t}$ where $x_{t}$ is defined by (R). We will show that $x_{n} \rightarrow Q(f)$.

We proceed with the following steps.

Step 1. We show that $\left\|x_{n}-z\right\| \leq \max \left\{\left\|x_{0}-z\right\|,(1 /(1-k))\|f(z)-z\|\right\}$ for all $n \geq 0$ and all $z \in F(T)$ and so $\left\{x_{n}\right\},\left\{y_{n}\right\},\left\{z_{n}\right\},\left\{f\left(x_{n}\right)\right\},\left\{T x_{n}\right\}$, and $\left\{T z_{n}\right\}$ are bounded.

Indeed, let $z \in F(T)$. Then, noting that

$$
\left\|z_{n}-z\right\| \leq \gamma_{n}\left\|x_{n}-z\right\|+\left(1-\gamma_{n}\right)\left\|T x_{n}-z\right\| \leq\left\|x_{n}-z\right\|,
$$

we have

$$
\begin{aligned}
\left\|y_{n}-z\right\| & =\left\|\beta_{n} x_{n}+\left(1-\beta_{n}\right)\left(T z_{n}-z\right)\right\| \\
& \leq \beta_{n}\left\|x_{n}-z\right\|+\left(1-\beta_{n}\right)\left\|x_{n}-z\right\| \leq\left\|x_{n}-z\right\|,
\end{aligned}
$$


which yields that

$$
\begin{aligned}
\left\|x_{n+1}-z\right\| & =\left\|\alpha_{n}\left(f\left(x_{n}\right)-z\right)+\left(1-\alpha_{n}\right)\left(y_{n}-z\right)\right\| \\
& \leq \alpha_{n}\left(\left\|f\left(x_{n}\right)-f(z)\right\|+\|f(z)-z\|\right)+\left(1-\alpha_{n}\right)\left\|x_{n}-z\right\| \\
& \leq \alpha_{n} k\left\|x_{n}-z\right\|+\alpha_{n}\|f(z)-z\|+\left(1-\alpha_{n}\right)\left\|x_{n}-z\right\| \\
& =\left(1-(1-k) \alpha_{n}\right)\left\|x_{n}-z\right\|+\alpha_{n}\|f(z)-z\| \\
& \leq \max \left\{\left\|x_{n}-z\right\|, \frac{1}{1-k}\|f(z)-z\|\right\} .
\end{aligned}
$$

Using an induction, we obtain

$$
\left\|x_{n}-z\right\| \leq \max \left\{\left\|x_{0}-z\right\|, \frac{1}{1-k}\|f(z)-z\|\right\}
$$

for all $n \geq 0$. Hence $\left\{x_{n}\right\}$ is bounded, and so are $\left\{y_{n}\right\},\left\{z_{n}\right\},\left\{T x_{n}\right\},\left\{T z_{n}\right\}$, and $\left\{f\left(x_{n}\right)\right\}$.

Step 2. We show that $\lim _{n} \rightarrow \infty\left\|x_{n+1}-y_{n}\right\|=0$ and $\lim _{n \rightarrow \infty}\left\|y_{n}-T z_{n}\right\|=0$. Indeed, it follows from condition (C1) and (C2) that

$$
\begin{aligned}
\left\|x_{n+1}-y_{n}\right\| & =\alpha_{n}\left\|f\left(x_{n}\right)-y_{n}\right\| \longrightarrow 0 \quad(\text { as } n \longrightarrow \infty) \\
\left\|y_{n}-T z_{n}\right\| & =\beta_{n}\left\|x_{n}-T z_{n}\right\| \longrightarrow 0 \quad(\text { as } n \longrightarrow \infty) .
\end{aligned}
$$

Also from $\lim _{n \rightarrow \infty}\left\|T z_{n}-z_{n}\right\|=0$, we get

$$
\left\|y_{n}-z_{n}\right\| \leq\left\|y_{n}-T z_{n}\right\|+\left\|T z_{n}-z_{n}\right\| \longrightarrow 0 \quad(\text { as } n \longrightarrow \infty)
$$

Step 3. We show that $\lim _{n \rightarrow \infty}\left\|x_{n+1}-x_{n}\right\|=0$. To this end, set $\delta_{n}=\left(1-\alpha_{n}\right) \gamma_{n}$ for $n \geq 0$. Then it follows from (C1) and (C3) that

$$
0<\liminf _{n \rightarrow \infty} \delta_{n} \leq \limsup _{n \rightarrow \infty} \delta_{n}<1
$$

Define

$$
x_{n+1}=\delta_{n} x_{n}+\left(1-\delta_{n}\right) w_{n}
$$


Observe that

$$
\begin{aligned}
w_{n+1}-w_{n}= & \frac{x_{n+2}-\delta_{n+1} x_{n+1}}{1-\delta_{n+1}}-\frac{x_{n+1}-\delta_{n} x_{n}}{1-\delta_{n}} \\
= & \frac{\alpha_{n+1} f\left(x_{n+1}\right)+\left(1-\alpha_{n+1}\right) y_{n+1}-\delta_{n+1} x_{n+1}}{1-\delta_{n+1}}-\frac{\alpha_{n} f\left(x_{n}\right)+\left(1-\alpha_{n}\right) y_{n}-\delta_{n} x_{n}}{1-\delta_{n}} \\
= & \left(\frac{\alpha_{n+1} f\left(x_{n+1}\right)}{1-\delta_{n+1}}-\frac{\alpha_{n} f\left(x_{n}\right)}{1-\delta_{n}}\right) \\
& +\frac{\left(1-\alpha_{n+1}\right)\left[\gamma_{n+1} x_{n+1}+\left(1-\gamma_{n+1}\right) T x_{n+1}\right]-\delta_{n+1} x_{n+1}+\left(1-\alpha_{n+1}\right)\left(y_{n+1}-z_{n+1}\right)}{1-\delta_{n+1}} \\
& -\frac{\left(1-\alpha_{n}\right)\left[\gamma_{n} x_{n}+\left(1-\gamma_{n}\right) T x_{n}\right]-\delta_{n} x_{n}+\left(1-\alpha_{n}\right)\left(y_{n}-z_{n}\right)}{1-\delta_{n}} \\
= & \left(\frac{\alpha_{n+1} f\left(x_{n+1}\right)}{1-\delta_{n+1}}-\frac{\alpha_{n} f\left(x_{n}\right)}{1-\delta_{n}}\right)+\frac{\left(1-\alpha_{n+1}\right)\left(1-\gamma_{n+1}\right) T x_{n+1}+\left(1-\alpha_{n+1}\right)\left(y_{n+1}-z_{n+1}\right)}{1-\delta_{n+1}} \\
& -\frac{\left(1-\alpha_{n}\right)\left(1-\gamma_{n}\right) T x_{n}+\left(1-\alpha_{n}\right)\left(y_{n}-z_{n}\right)}{1-\delta_{n}} \\
= & \left(\frac{\alpha_{n+1} f\left(x_{n+1}\right)}{1-\delta_{n+1}}-\frac{\alpha_{n} f\left(x_{n}\right)}{1-\delta_{n}}\right)+\left(T x_{n+1}-T x_{n}\right)-\frac{\alpha_{n+1}}{1-\delta_{n+1}} T x_{n+1}+\frac{\alpha_{n}}{1-\delta_{n}} T x_{n} \\
& +\frac{\left(1-\alpha_{n+1}\right)\left(y_{n+1}-z_{n+1}\right)}{1-\delta_{n+1}}-\frac{\left(1-\alpha_{n}\right)\left(y_{n}-z_{n}\right)}{1-\delta_{n}} .
\end{aligned}
$$

It follows from (3.13) that

$$
\begin{aligned}
& \left\|w_{n+1}-w_{n}\right\|-\left\|x_{n+1}-x_{n}\right\| \\
& \quad \leq \frac{\alpha_{n+1}}{1-\delta_{n+1}}\left(\left\|f\left(x_{n+1}\right)\right\|+\left\|T x_{n+1}\right\|\right)+\frac{\alpha_{n}}{1-\delta_{n}}\left(\left\|f\left(x_{n}\right)\right\|+\left\|T x_{n}\right\|\right) \\
& \quad+\frac{1-\alpha_{n+1}}{1-\delta_{n+1}}\left\|y_{n+1}-z_{n+1}\right\|+\frac{1-\alpha_{n}}{1-\delta_{n}}\left\|y_{n}-z_{n}\right\| .
\end{aligned}
$$

Since $\left\{f\left(x_{n}\right)\right\}$ and $\left\{T x_{n}\right\}$ are bounded, by (C1), (3.10), (3.11), and (3.14) we obtain that

$$
\limsup _{n \rightarrow \infty}\left(\left\|w_{n+1}-w_{n}\right\|-\left\|x_{n+1}-x_{n}\right\|\right) \leq 0
$$

Hence by Lemma 2.2, we have

$$
\lim _{n \rightarrow \infty}\left\|w_{n}-x_{n}\right\|=0
$$


It follows from (3.11) and (3.12) that

$$
\lim _{n \rightarrow \infty}\left\|x_{n+1}-x_{n}\right\|=0
$$

Step 4. We show that $\lim _{n \rightarrow \infty}\left\|x_{n}-T x_{n}\right\|=0$. In fact, from (IS) it follows that

$$
\begin{aligned}
\left\|T x_{n}-x_{n}\right\| & \leq\left\|x_{n}-x_{n+1}\right\|+\left\|x_{n+1}-y_{n}\right\|+\left\|y_{n}-T z_{n}\right\|+\left\|T z_{n}-T x_{n}\right\| \\
& \leq\left\|x_{n}-x_{n+1}\right\|+\left\|x_{n+1}-y_{n}\right\|+\left\|y_{n}-T z_{n}\right\|+\left(1-\gamma_{n}\right)\left\|T x_{n}-x_{n}\right\| .
\end{aligned}
$$

So we have

$$
\gamma_{n}\left\|T x_{n}-x_{n}\right\| \leq\left\|x_{n}-x_{n+1}\right\|+\left\|x_{n+1}-y_{n}\right\|+\left\|y_{n}-T z_{n}\right\| .
$$

Thus, from condition (C3), Steps 2, and 3, we have

$$
\lim _{n \rightarrow \infty}\left\|T x_{n}-x_{n}\right\|=0
$$

Step 5. We show that $\lim \sup _{n \rightarrow \infty}\left\langle Q(f)-f(Q(f)), J\left(Q(f)-x_{n}\right)\right\rangle \leq 0$. To prove this, let a subsequence $\left\{x_{n_{j}}\right\}$ of $\left\{x_{n}\right\}$ be such that

$$
\limsup _{n \rightarrow \infty}\left\langle Q(f)-f(Q(f)), J\left(Q(f)-x_{n}\right)\right\rangle=\lim _{j \rightarrow \infty}\left\langle Q(f)-f(Q(f)), J\left(Q(f)-x_{n_{j}}\right)\right\rangle
$$

and $x_{n_{j}} \rightarrow q$ for some $q \in E$. From Step 4, it follows that $\lim _{j \rightarrow \infty}\left\|x_{n_{j}}-T x_{n_{j}}\right\|=0$.

Now let $Q(f)=\lim _{t \rightarrow 0^{+}} x_{t}$, where $x_{t}=t f\left(x_{t}\right)+(1-t) T x_{t}$. Then we can write

$$
x_{t}-x_{n_{j}}=t\left(f\left(x_{t}\right)-x_{n_{j}}\right)+(1-t)\left(T x_{t}-x_{n_{j}}\right) .
$$

\section{Putting}

$$
a_{j}(t)=(1-t)^{2}\left\|T x_{n_{j}}-x_{n_{j}}\right\|\left(2\left\|x_{t}-x_{n_{j}}\right\|+\left\|T x_{n_{j}}-x_{n_{j}}\right\|\right) \longrightarrow 0 \quad(j \rightarrow \infty)
$$


by Step 4 and using Lemma 2.1, we obtain

$$
\begin{aligned}
\left\|x_{t}-x_{n_{j}}\right\|^{2} \leq & (1-t)^{2}\left\|T x_{t}-x_{n_{j}}\right\|^{2}+2 t\left\langle f\left(x_{t}\right)-x_{n_{j}} J\left(x_{t}-x_{n_{j}}\right)\right\rangle \\
\leq & (1-t)^{2}\left(\left\|T x_{t}-T x_{n_{j}}\right\|+\left\|T x_{n_{j}}-x_{n_{j}}\right\|\right)^{2} \\
& +2 t\left\langle f\left(x_{t}\right)-x_{t}, J\left(x_{t}-x_{n_{j}}\right)\right\rangle+2 t\left\|x_{t}-x_{n_{j}}\right\|^{2} \\
\leq & (1-t)^{2}\left\|x_{t}-x_{n_{j}}\right\|^{2}+a_{j}(t) \\
& +2 t\left\langle f\left(x_{t}\right)-x_{t}, J\left(x_{t}-x_{n_{j}}\right)\right\rangle+2 t\left\|x_{t}-x_{n_{j}}\right\|^{2} .
\end{aligned}
$$

The last inequality implies

$$
\left\langle x_{t}-f\left(x_{t}\right), J\left(x_{t}-x_{n_{j}}\right)\right\rangle \leq \frac{t}{2}\left\|x_{t}-x_{n_{j}}\right\|^{2}+\frac{1}{2 t} a_{j}(t) .
$$

It follows that

$$
\limsup _{j \rightarrow \infty}\left\langle x_{t}-f\left(x_{t}\right), J\left(x_{t}-x_{n_{j}}\right)\right\rangle \leq \frac{t}{2} M
$$

where $M>0$ is a constant such that $M \geq\left\|x_{t}-x_{n}\right\|^{2}$ for all $n \geq 0$ and $t \in(0,1)$. Taking the $\limsup$ as $t \rightarrow 0$ in (3.26) and noticing the fact that the two limits are interchangeable due to the fact that $J$ is uniformly continuous on bounded subsets of $E$ from the strong topology of $E$ to the weak* topology of $E^{*}$, we have

$$
\limsup _{j \rightarrow \infty}\left\langle Q(f)-f(Q(f)), J\left(Q(f)-x_{n_{j}}\right)\right\rangle \leq 0
$$

Indeed, letting $t \rightarrow 0$, from (3.26) we have

$$
\limsup _{t \rightarrow 0} \limsup _{j \rightarrow \infty}\left\langle x_{t}-f\left(x_{t}\right), J\left(x_{t}-x_{n_{j}}\right)\right\rangle \leq 0
$$

So, for any $\varepsilon>0$, there exists a positive number $\delta_{1}$ such that for any $t \in\left(0, \delta_{1}\right)$,

$$
\limsup _{j \rightarrow \infty}\left\langle x_{t}-f\left(x_{t}\right), J\left(x_{t}-x_{n_{j}}\right)\right\rangle \leq \frac{\varepsilon}{2}
$$


Moreover, since $x_{t} \rightarrow Q(f)$ as $t \rightarrow 0$, the set $\left\{x_{t}-x_{n_{j}}\right\}$ is bounded and the duality mapping $J$ is norm-to-weak ${ }^{*}$ uniformly continuous on bounded subset of $E$, there exists $\delta_{2}>0$ such that, for any $t \in\left(0, \delta_{2}\right)$,

$$
\begin{aligned}
\mid\langle Q(f) & \left.-f(Q(f)), J\left(Q(f)-x_{n_{j}}\right)\right\rangle-\left\langle x_{t}-f\left(x_{t}\right), J\left(x_{t}-x_{n_{j}}\right)\right\rangle \mid \\
= & \mid\left\langle Q(f)-f(Q(f)), J\left(Q(f)-x_{n_{j}}\right)-J\left(x_{t}-x_{n_{j}}\right)\right\rangle \\
& +\left\langle Q(f)-f(Q(f))-\left(x_{t}-f\left(x_{t}\right)\right), J\left(x_{t}-x_{n_{j}}\right)\right\rangle \mid \\
\leq & \left|\left\langle Q(f)-f(Q(f)), J\left(x_{t}-x_{n_{j}}\right)-J\left(Q(f)-x_{n_{j}}\right)\right\rangle\right| \\
& +\left\|Q(f)-f(Q(f))-\left(x_{t}-f\left(x_{t}\right)\right)\right\|\left\|x_{t}-x_{n_{j}}\right\|<\frac{\varepsilon}{2} .
\end{aligned}
$$

Choose $\delta=\min \left\{\delta_{1}, \delta_{2}\right\}$, we have for all $t \in(0, \delta)$ and $j \in \mathbb{N}$,

$$
\left\langle Q(f)-f(Q(f)), J\left(Q(f)-x_{n_{j}}\right)\right\rangle\left\langle\left\langle x_{t}-f\left(x_{t}\right), J\left(x_{t}-x_{n_{j}}\right)\right\rangle+\frac{\varepsilon}{2}\right.
$$

which implies that

$$
\limsup _{j \rightarrow \infty}\left\langle Q(f)-f(Q(f)), J\left(Q(f)-x_{n_{j}}\right)\right\rangle \leq \limsup _{j \rightarrow \infty}\left\langle x_{t}-f\left(x_{t}\right), J\left(x_{t}-x_{n_{j}}\right)\right\rangle+\frac{\varepsilon}{2} .
$$

Since $\lim \sup _{j \rightarrow \infty}\left\langle x_{t}-f\left(x_{t}\right), J\left(x_{t}-x_{n_{j}}\right)\right\rangle \leq \varepsilon / 2$, we have

$$
\limsup _{j \rightarrow \infty}\left\langle Q(f)-f(Q(f)), J\left(Q(f)-x_{n_{j}}\right)\right\rangle \leq \varepsilon
$$

Since $\varepsilon$ is arbitrary, we obtain that

$$
\limsup _{j \rightarrow \infty}\left\langle Q(f)-f(Q(f)), J\left(Q(f)-x_{n_{j}}\right)\right\rangle \leq 0
$$

Step 6. We show that $\lim _{n \rightarrow \infty}\left\|x_{n}-Q(f)\right\|=0$. By using (IS), we have

$$
\left\|x_{n+1}-Q(f)\right\|=\left\|\alpha_{n}\left(f\left(x_{n}\right)-Q(f)\right)+\left(1-\alpha_{n}\right)\left(y_{n}-Q(f)\right)\right\| .
$$


Applying Lemma 2.1 and (3.6), we obtain

$$
\begin{aligned}
&\left\|x_{n+1}-Q(f)\right\|^{2} \\
& \leq\left(1-\alpha_{n}\right)^{2}\left\|y_{n}-Q(f)\right\|^{2}+2 \alpha_{n}\left\langle f\left(x_{n}\right)-Q(f), J\left(x_{n+1}-Q(f)\right)\right\rangle \\
& \leq\left(1-\alpha_{n}\right)^{2}\left\|x_{n}-Q(f)\right\|^{2}+2 \alpha_{n}\left\langle f\left(x_{n}\right)-f(Q(f)), J\left(x_{n+1}-Q(f)\right)\right\rangle \\
&+2 \alpha_{n}\left\langle f(Q(f))-Q(f), J\left(x_{n+1}-Q(f)\right)\right\rangle \\
& \leq\left(1-\alpha_{n}\right)^{2}\left\|x_{n}-Q(f)\right\|^{2}+2 k \alpha_{n}\left\|x_{n}-Q(f)\right\|\left\|x_{n+1}-Q(f)\right\| \\
&+2 \alpha_{n}\left\langle f(Q(f))-Q(f), J\left(x_{n+1}-Q(f)\right)\right\rangle \\
& \leq\left(1-2 \alpha_{n}+\alpha_{n}^{2}\right)\left\|x_{n}-Q(f)\right\|^{2}+k \alpha_{n}\left(\left\|x_{n}-Q(f)\right\|^{2}+\left\|x_{n+1}-Q(f)\right\|^{2}\right) \\
&+2 \alpha_{n}\left\langle f(Q(f))-Q(f), J\left(x_{n+1}-Q(f)\right)\right\rangle .
\end{aligned}
$$

It then follows that

$$
\begin{aligned}
\left\|x_{n+1}-Q(f)\right\|^{2} & \\
\leq & \left(1-\frac{2(1-k) \alpha_{n}}{1-k \alpha_{n}}\right)\left\|x_{n}-Q(f)\right\|^{2}+\frac{\alpha_{n}^{2}}{1-k \alpha_{n}}\left\|x_{n}-Q(f)\right\|^{2} \\
& +\frac{2 \alpha_{n}}{1-k \alpha_{n}}\left\langle Q(f)-f(Q(f)), J\left(Q(f)-x_{n+1}\right)\right\rangle \\
\leq & \left(1-\frac{2(1-k) \alpha_{n}}{1-k \alpha_{n}}\right)\left\|x_{n}-Q(f)\right\|^{2}+\frac{\alpha_{n}^{2}}{1-k \alpha_{n}} M^{2} \\
& +\frac{2 \alpha_{n}}{1-k \alpha_{n}}\left\langle Q(f)-f(Q(f)), J\left(Q(f)-x_{n+1}\right)\right\rangle,
\end{aligned}
$$

where $M=\sup _{n \geq 0}\left\|x_{n}-Q(f)\right\|$. Put

$$
\begin{aligned}
& \lambda_{n}=\frac{2(1-k) \alpha_{n}}{1-k \alpha_{n}} \\
& \gamma_{n}=\frac{\alpha_{n}}{2(1-k)} M^{2}+\frac{1}{1-k}\left\langle Q(f)-f(Q(f)), J\left(Q(f)-x_{n+1}\right)\right\rangle .
\end{aligned}
$$

From the condition (C1) and Step 5, it follows that $\lambda_{n} \rightarrow 0, \sum_{n=0}^{\infty} \lambda_{n}=\infty$, and $\lim \sup _{n \rightarrow \infty} \gamma_{n} \leq$ 0 . Since (3.37) reduces to

$$
\left\|x_{n+1}-Q(f)\right\|^{2} \leq\left(1-\lambda_{n}\right)\left\|x_{n}-Q(f)\right\|^{2}+\lambda_{n} \gamma_{n}
$$

from Lemma 2.3 with $\delta_{n}=0$, we conclude that $\lim _{n \rightarrow \infty}\left\|x_{n}-Q(f)\right\|=0$. This completes the proof. 
Corollary 3.3. Let E be a uniformly smooth Banach space. Let $C, T,\left\{\alpha_{n}\right\},\left\{\beta_{n}\right\},\left\{\gamma_{n}\right\}, f, x_{0},\left\{z_{n}\right\}$, $\left\{y_{n}\right\}$, and $\left\{x_{n}\right\}$ be the same as in Theorem 3.2. Then the conclusion of Theorem 3.2 still holds.

Proof. Since $E$ is a uniformly smooth Banach space, $E$ is reflexive, the norm is uniformly Gâteaux differentiable, and every nonempty weakly compact convex subset of $E$ has the fixed point property for nonexpansive mappings. Thus the conclusion of Corollary 3.3 follows from Theorem 3.2 immediately.

Corollary 3.4. Let $C$ be a nonempty closed convex subset of a uniformly smooth Banach space $E$. Let $T: C \rightarrow C$ be a nonexpansive mapping with $F(T) \neq \emptyset$. Let $\left\{\alpha_{n}\right\},\left\{\beta_{n}\right\}$, and $\left\{\gamma_{n}\right\}$ be three sequences in $(0,1)$ which satisfy the control conditions $(C 1)-(C 3)$ in Theorem 3.2. Then for the initial guess $x_{0} \in C$ and $u \in C$, the sequence $\left\{x_{n}\right\}$ generated by (1.6) converges strongly to a fixed point of $T$ under the assumption $\lim _{n \rightarrow \infty}\left\|T z_{n}-z_{n}\right\|=0$.

Remark 3.5. (1) In general, the condition (C3) in Theorem 3.2 and the condition $\sum_{n=0}^{\infty} \mid \gamma_{n+1}-$ $\gamma_{n} \mid<\infty$ of Qin et al. [16, Theorem 2.1] are not comparable; neither of them implies the other. Theorem 3.2 (and Corollary 3.4) removes the conditions $\sum_{n=0}^{\infty}\left|\alpha_{n+1}-\alpha_{n}\right|<\infty$ and $\sum_{n=0}^{\infty} \mid \beta_{n+1}-$ $\beta_{n} \mid<\infty$ imposed on the control parameters $\left\{\alpha_{n}\right\}$ and $\left\{\beta_{n}\right\}$ of Qin et al. [16, Theorem 2.1].

(2) Theorem 3.2 (and Corollary 3.3) complements the corresponding results in Moudafi [11], $\mathrm{Xu}$ [12], and Yao et al. [15]. In particular, if $\beta_{n}=0$ in (IS), then (IS) in Theorem 3.2 reduces a new one for finding a fixed point of $T$ :

$$
\begin{aligned}
z_{n} & =\gamma_{n} x_{n}+\left(1-\gamma_{n}\right) T x_{n}, \\
x_{n+1} & =\alpha_{n} f\left(x_{n}\right)+\left(1-\alpha_{n}\right) T z_{n}, \quad n \geq 0 .
\end{aligned}
$$

(3) Corollary 3.4 with $\beta_{n}=0$ in (IS) develops the corresponding results of Shioji and Takahashi [8], Wittmann [9] without the condition $\sum_{n=0}^{\infty}\left|\alpha_{n+1}-\alpha_{n}\right|<\infty$ as well as the result of Chang [2] in which the condition $\lim _{n \rightarrow \infty}\left\|T x_{n}-x_{n}\right\|=0$ was assumed.

Next, we consider the viscosity iterative scheme with the weakly contractive mappings instead of the contractions.

Theorem 3.6. Let E be a reflexive Banach space having a uniformly Gâteaux differentiable norm. Suppose that every weakly compact convex subset of $E$ has the fixed point property for nonexpansive mappings. Let $C$ be a nonempty closed convex subset of $E$ and let $T$ be a nonexpansive mapping from $C$ into itself with $F(T) \neq \emptyset$. Let $\left\{\alpha_{n}\right\},\left\{\beta_{n}\right\}$, and $\left\{\gamma_{n}\right\}$ be sequences in $(0,1)$ which satisfy the conditions (C1)-(C3) in Theorem 3.2. Let $A: C \rightarrow C$ be a weakly contractive mapping and let $x_{0} \in C$ be chosen arbitrarily. Let $\left\{x_{n}\right\}$ be the sequence generated by

$$
\begin{aligned}
z_{n} & =\gamma_{n} x_{n}+\left(1-\gamma_{n}\right) T x_{n}, \\
y_{n} & =\beta_{n} x_{n}+\left(1-\beta_{n}\right) T z_{n}, \\
x_{n+1} & =\alpha_{n} A x_{n}+\left(1-\alpha_{n}\right) y_{n}, \quad n \geq 0 .
\end{aligned}
$$

If $\lim _{n \rightarrow \infty}\left\|T z_{n}-z_{n}\right\|=0$, then $\left\{x_{n}\right\}$ converges strongly to $Q\left(A x^{*}\right)=x^{*} \in F(T)$, where $Q$ is a sunny nonexpansive retraction from $C$ onto $F(T)$. 
Proof. It follows from Remark 3.1 that $F(T)$ is the sunny nonexpansive retract of $C$. Denote by $Q$ the sunny nonexpansive retraction of $C$ onto $F(T)$. Then $Q \circ A$ is a weakly contractive mapping of $C$ into itself. Indeed,

$$
\|Q(A x)-Q(A y)\| \leq\|A x-A y\| \leq\|x-y\|-\psi(\|x-y\|), \quad \forall x, y \in C
$$

Lemma 2.4 assures that there exists a unique element $x^{*} \in C$ such that $x^{*}=Q\left(A x^{*}\right)$. Such a $x^{*} \in C$ is an element of $F(T)$.

Now we define an iterative scheme as follows:

$$
\begin{aligned}
u_{n} & =\gamma_{n} w_{n}+\left(1-\gamma_{n}\right) T w_{n}, \\
v_{n} & =\beta_{n} w_{n}+\left(1-\beta_{n}\right) T u_{n}, \\
w_{n+1} & =\alpha_{n} A x^{*}+\left(1-\alpha_{n}\right) v_{n}, \quad n \geq 0 .
\end{aligned}
$$

Let $\left\{w_{n}\right\}$ be the sequence generated by (3.37). Then, by taking $u_{n}$ as $z_{n}$ in $\lim _{n \rightarrow \infty}\left\|T z_{n}-z_{n}\right\|=$ 0 , Theorem 3.2 with $f=A x^{*}$ a constant assures that $\left\{w_{n}\right\}$ converges strongly to $Q\left(A x^{*}\right)=x^{*}$ as $n \rightarrow \infty$. For any $n \geq 0$, observe that

$$
\begin{aligned}
\left\|y_{n}-v_{n}\right\| & \leq \beta_{n}\left\|x_{n}-w_{n}\right\|+\left(1-\beta_{n}\right)\left\|T z_{n}-T u_{n}\right\| \\
& \leq \beta_{n}\left\|x_{n}-w_{n}\right\|+\left(1-\beta_{n}\right)\left\|z_{n}-u_{n}\right\| \\
& \leq \beta_{n}\left\|x_{n}-w_{n}\right\|+\left(1-\beta_{n}\right)\left\|\gamma_{n} x_{n}+\left(1-\gamma_{n}\right) T x_{n}-\left(\gamma_{n} w_{n}+\left(1-\gamma_{n}\right) T w_{n}\right)\right\| \\
& \leq \beta_{n}\left\|x_{n}-w_{n}\right\|+\left(1-\beta_{n}\right)\left[\gamma_{n}\left\|x_{n}-w_{n}\right\|+\left(1-\gamma_{n}\right)\left\|x_{n}-w_{n}\right\|\right] \\
& =\left\|x_{n}-w_{n}\right\|
\end{aligned}
$$

Then we have

$$
\begin{aligned}
\left\|x_{n+1}-w_{n+1}\right\| \leq & \alpha_{n}\left\|A x_{n}-A x^{*}\right\|+\left(1-\alpha_{n}\right)\left\|y_{n}-v_{n}\right\| \\
\leq & \alpha_{n}\left(\left\|A x_{n}-A w_{n}\right\|+\left\|A w_{n}-A x^{*}\right\|\right)+\left(1-\alpha_{n}\right)\left\|x_{n}-w_{n}\right\| \\
\leq & \alpha_{n}\left\|x_{n}-w_{n}\right\|-\alpha_{n} \psi\left(\left\|x_{n}-w_{n}\right\|\right)+\alpha_{n}\left(\left\|w_{n}-x^{*}\right\|-\psi\left(\left\|w_{n}-x^{*}\right\|\right)\right) \\
& \quad+\left(1-\alpha_{n}\right)\left\|x_{n}-w_{n}\right\| \\
\leq & \left\|x_{n}-w_{n}\right\|-\alpha_{n} \psi\left(\left\|x_{n}-w_{n}\right\|\right)+\alpha_{n}\left\|w_{n}-x^{*}\right\| .
\end{aligned}
$$

Thus, for $s_{n}=\left\|x_{n}-w_{n}\right\|$, we obtain the following recursive inequality:

$$
s_{n+1} \leq s_{n}-\alpha_{n} \psi\left(s_{n}\right)+\alpha_{n}\left\|w_{n}-x^{*}\right\|
$$


Since $\left\|w_{n}-x^{*}\right\| \rightarrow 0$, it follows from Lemma 2.5 that $\lim _{n \rightarrow \infty}\left\|x_{n}-w_{n}\right\|=0$. Hence

$$
\lim _{n \rightarrow \infty}\left\|x_{n}-x^{*}\right\| \leq \lim _{n \rightarrow \infty}\left(\left\|x_{n}-w_{n}\right\|+\left\|w_{n}-x^{*}\right\|\right)=0 .
$$

This completes the proof.

Corollary 3.7. Let $E$ be a uniformly smooth Banach space. Let $C, T, A, x_{0},\left\{\alpha_{n}\right\},\left\{\beta_{n}\right\},\left\{\gamma_{n}\right\},\left\{z_{n}\right\}$, $\left\{y_{n}\right\}$, and $\left\{x_{n}\right\}$ be the same as in Theorem 3.6. Then the conclusion of Theorem 3.6 still holds.

Remark 3.8. (1) Theorem 3.6 (and Corollary 3.7) develops and complements the corresponding results in Moudafi [11], Qin et al. [16], Shioji and Takahashi [8], Wittmann [9], Xu [10, 12], and Yao et al. [15].

(2) Even in the case of $\beta_{n}=0$ in Theorem 3.6, Theorem 3.6 appears to be independent of Theorem 5.6 of Wong et al. [13] in which the control conditions (C1) and $\sum_{n=0}^{\infty}\left|\alpha_{n+1}-\alpha_{n}\right|<\infty$ were utilized. In fact, it appears to be unknown whether a reflexive and strictly convex space satisfies the fixed point property for nonexpansive mappings.

(3) The merits of our results in this paper are that fewer restrictions are imposed on the control parameters $\left\{\alpha_{n}\right\},\left\{\beta_{n}\right\}$, and $\left\{\gamma_{n}\right\}$. All of our results can be viewed as a supplement to the results obtained by Qin et al. [16], Kim and Xu [14], and Yao et al. [15].

(4) The conclusions of Theorems 3.2 and 3.6 still hold if $E$ is assumed to be strictly convex instead of having the fixed point property for nonexpansive mappings.

\section{Acknowledgments}

The author thanks the editor Professor Simeon Reich and the referees for their careful reading and valuable suggestions to improve the writing of this paper.

\section{References}

[1] F. E. Browder, "Convergence of approximants to fixed points of nonexpansive non-linear mappings in Banach spaces," Archive for Rational Mechanics and Analysis, vol. 24, no. 1, pp. 82-90, 1967.

[2] S. S. Chang, “On Halpern's open question," Acta Mathematica Sinica, vol. 48, no. 5, pp. 979-984, 2005.

[3] Y. J. Cho, S. M. Kang, and H. Zhou, "Some control conditions on iterative methods," Communications on Applied Nonlinear Analysis, vol. 12, no. 2, pp. 27-34, 2005.

[4] B. Halpern, "Fixed points of nonexpanding maps," Bulletin of the American Mathematical Society, vol. 73, no. 6, pp. 957-961, 1967.

[5] P.-L. Lions, "Approximation de points fixes de contractions," Comptes Rendus de l'Académie des Sciences. Série A-B, vol. 284, no. 21, pp. 1357-1359, 1977.

[6] S. Reich, "Strong convergence theorems for resolvents of accretive operators in Banach spaces," Journal of Mathematical Analysis and Applications, vol. 75, no. 1, pp. 287-292, 1980.

[7] S. Reich, "Approximating fixed points of nonexpansive mappings," PanAmerican Mathematical Journal, vol. 4, no. 2, pp. 23-28, 1994.

[8] N. Shioji and W. Takahashi, "Strong convergence of approximated sequences for nonexpansive mappings in Banach spaces," Proceedings of the American Mathematical Society, vol. 125, no. 12, pp. 3641-3645, 1997.

[9] R. Wittmann, "Approximation of fixed points of nonexpansive mappings," Archiv der Mathematik, vol. 58 , no. 5, pp. 486-491, 1992.

[10] H.-K. Xu, "Iterative algorithms for nonlinear operators," Journal of the London Mathematical Society, vol. 66, no. 1, pp. 240-256, 2002. 
[11] A. Moudafi, "Viscosity approximation methods for fixed-points problems," Journal of Mathematical Analysis and Applications, vol. 241, no. 1, pp. 46-55, 2000.

[12] H.-K. Xu, "Viscosity approximation methods for nonexpansive mappings," Journal of Mathematical Analysis and Applications, vol. 298, no. 1, pp. 279-291, 2004.

[13] N. C. Wong, D. R. Sahu, and J. C. Yao, “Solving variational inequalities involving nonexpansive type mappings," Nonlinear Analysis: Theory, Methods E Applications, vol. 69, no. 12, pp. 4732-4753, 2008.

[14] T.-H. Kim and H.-K. Xu, "Strong convergence of modified Mann iterations," Nonlinear Analysis: Theory, Methods \& Applications, vol. 61, no. 1-2, pp. 51-60, 2005.

[15] Y. Yao, R. Chen, and J.-C. Yao, "Strong convergence and certain control conditions for modified Mann iteration," Nonlinear Analysis: Theory, Methods \& Applications, vol. 68, no. 6, pp. 1687-1693, 2008.

[16] X. Qin, Y. Su, and M. Shang, "Strong convergence of the composite Halpern iteration," Journal of Mathematical Analysis and Applications, vol. 339, no. 2, pp. 996-1002, 2008.

[17] I. Cioranescu, Geometry of Banach Spaces, Duality Mappings and Nonlinear Problems, vol. 62 of Mathematics and Its Applications, Kluwer Academic Publishers, Dordrecht, The Netherlands, 1990.

[18] K. Goebel and S. Reich, Uniform Convexity, Hyperbolic Geometry, and Nonexpansive Mappings, vol. 83 of Monographs and Textbooks in Pure and Applied Mathematics, Marcel Dekker, New York, NY, USA, 1984.

[19] J. S. Jung and C. H. Morales, "The Mann process for perturbed $m$-accretive operators in Banach spaces," Nonlinear Analysis: Theory, Methods E Applications, vol. 46, no. 2, pp. 231-243, 2001.

[20] T. Suzuki, "Strong convergence of Krasnoselskii and Mann's type sequences for one-parameter nonexpansive semigroups without Bochner integrals," Journal of Mathematical Analysis and Applications, vol. 305, no. 1, pp. 227-239, 2005.

[21] L. S. Liu, "Ishikawa and Mann iterative process with errors for nonlinear strongly accretive mappings in Banach spaces," Journal of Mathematical Analysis and Applications, vol. 194, no. 1, pp. 114-125, 1995.

[22] B. E. Rhoades, "Some theorems on weakly contractive maps," Nonlinear Analysis: Theory, Methods $\mathcal{E}$ Applications, vol. 47, no. 4, pp. 2683-2693, 2001.

[23] Ya. I. Alber and A. N. Iusem, "Extension of subgradient techniques for nonsmooth optimization in Banach spaces," Set-Valued Analysis, vol. 9, no. 4, pp. 315-335, 2001.

[24] Ya. I. Alber, S. Reich, and J.-C. Yao, "Iterative methods for solving fixed-point problems with nonselfmappings in Banach spaces," Abstract and Applied Analysis, vol. 2003, no. 4, pp. 193-216, 2003.

[25] J. S. Jung, "Viscosity approximation methods for a family of finite nonexpansive mappings in Banach spaces," Nonlinear Analysis: Theory, Methods E Applications, vol. 64, no. 11, pp. 2536-2552, 2006. 


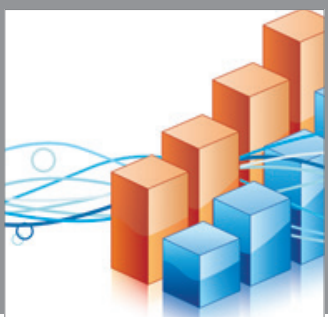

Advances in

Operations Research

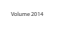

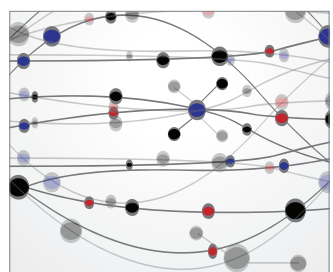

\section{The Scientific} World Journal
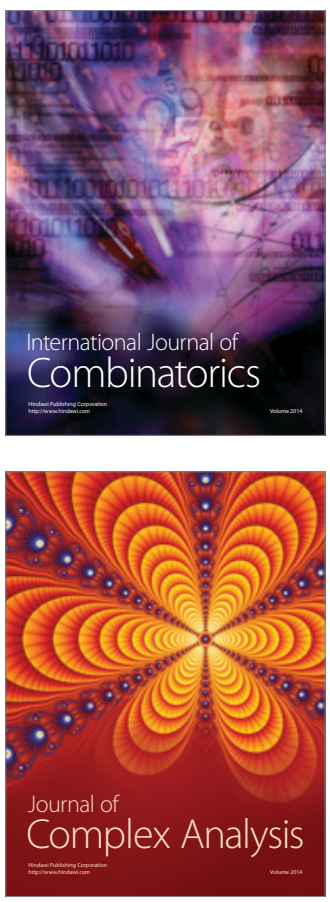

International Journal of

Mathematics and

Mathematical

Sciences
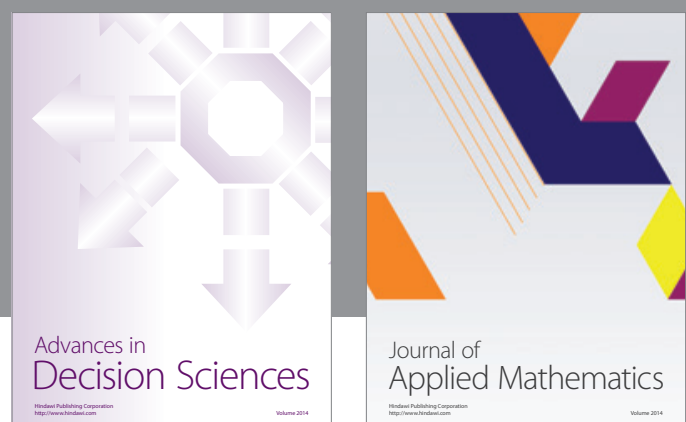

Journal of

Applied Mathematics
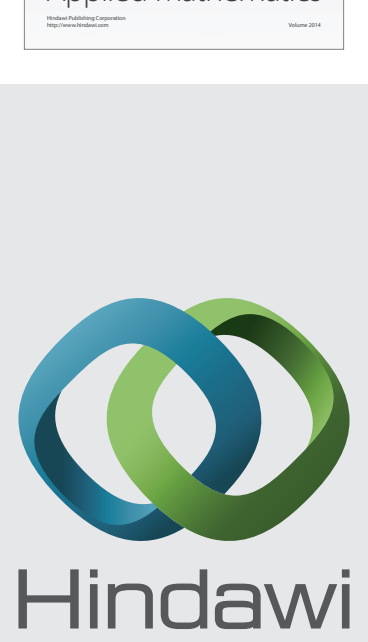

Submit your manuscripts at http://www.hindawi.com
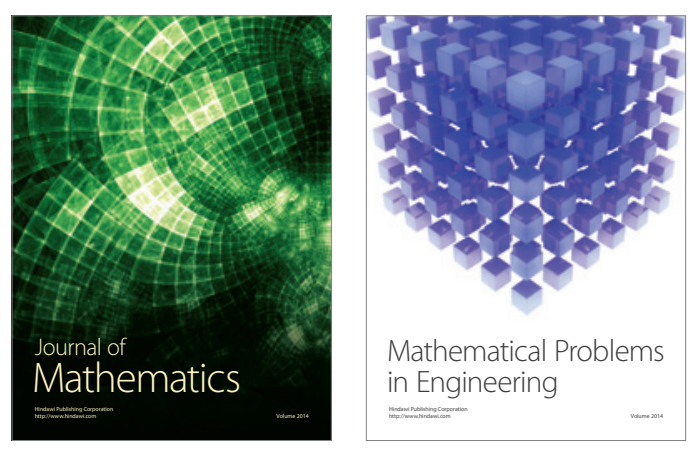

Mathematical Problems in Engineering
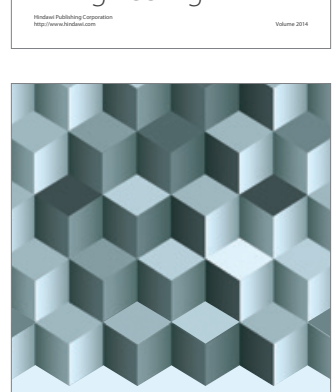

Journal of

Function Spaces
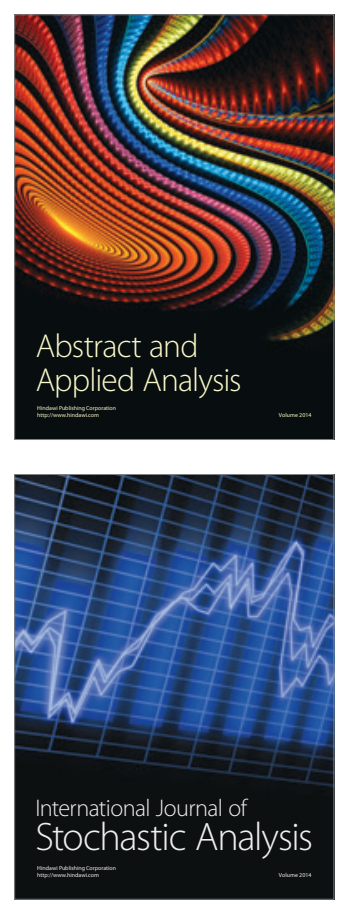

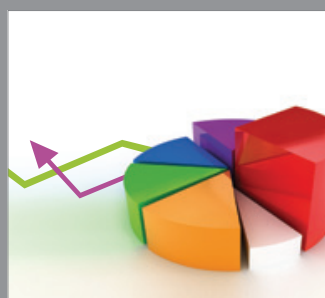

ournal of

Probability and Statistics

Promensencen
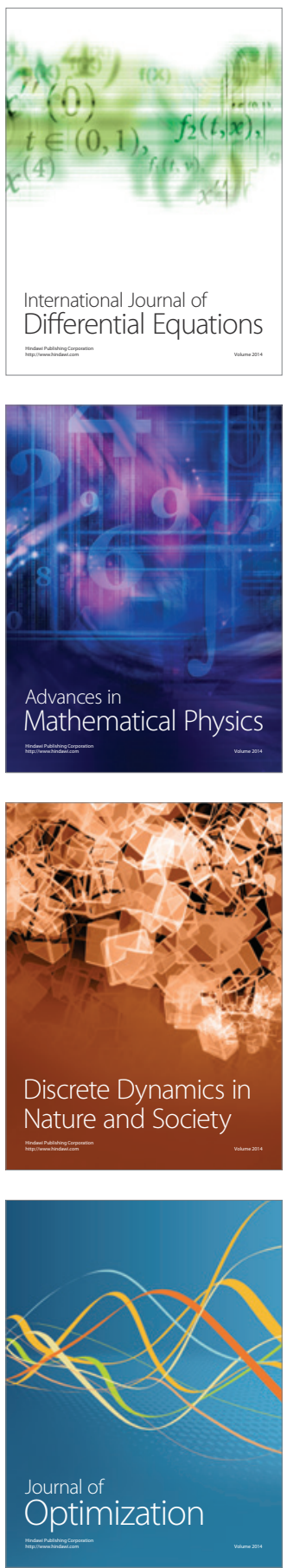